\title{
Inhomogeneous Gilbert damping from impurities and electron-electron interactions
}

\author{
E. M. Hankiewicz, ${ }^{1,2, *}$ G. Vignale, ${ }^{2}$ and Y. Tserkovnyak ${ }^{3}$ \\ ${ }^{1}$ Department of Physics, Fordham University, Bronx, New York 10458, USA \\ ${ }^{2}$ Department of Physics and Astronomy, University of Missouri, Columbia, Missouri 65211, USA \\ ${ }^{3}$ Department of Physics and Astronomy, University of California, Los Angeles, California 90095, USA \\ (Received 7 April 2008; revised manuscript received 3 June 2008; published 22 July 2008)
}

\begin{abstract}
We present a unified theory of magnetic damping in itinerant electron ferromagnets at order $q^{2}$ including electron-electron interactions and disorder scattering. We show that the Gilbert damping coefficient can be expressed in terms of the spin conductivity, leading to a Matthiessen-type formula in which disorder and interaction contributions are additive. In a weak ferromagnet regime, electron-electron interactions lead to a strong enhancement of the Gilbert damping.
\end{abstract}

DOI: 10.1103/PhysRevB.78.020404

PACS number(s): 76.50.+g, 75.45. $+\mathrm{j}$, 75.30.Ds

\section{INTRODUCTION}

In spite of much effort, a complete theoretical description of the damping of ferromagnetic spin waves in itinerant electron ferromagnets is not yet available. ${ }^{1}$ Recent measurements of the dispersion and damping of spin-wave excitations driven by a direct spin-polarized current prove that the theoretical picture is incomplete, particularly when it comes to calculating the linewidth of these excitations. ${ }^{2}$ One of the most important parameters of the theory is the so-called Gilbert damping parameter $\alpha,{ }^{3}$ which controls the damping rate and thermal noise and is often assumed to be independent of the wave vector of the excitations. This assumption is justified for excitations of very long wavelength (e.g., a homogeneous precession of the magnetization), where $\alpha$ can originate in a relatively weak spin-orbit (SO) interaction. ${ }^{4}$ However, it becomes dubious as the wave vector $q$ of the excitations grows. Indeed, both electron-electron $(e-e)$ and electron-impurity interactions can cause an inhomogeneous magnetization to decay into spin-flipped electron-hole pairs, giving rise to a $q^{2}$ contribution to the Gilbert damping. In practice, the presence of this contribution means that the Landau-Lifshitz-Gilbert equation contains a term proportional to $-\mathbf{m} \times \nabla^{2} \partial_{t} \mathbf{m}$ (where $\mathbf{m}$ is the magnetization) and requires neither spin-orbit nor magnetic disorder scattering. In contrast, the homogeneous damping term is of the form $\mathbf{m} \times \partial_{t} \mathbf{m}$ and vanishes in the absence of SO or magnetic disorder scattering.

The influence of disorder on the linewidth of spin waves in itinerant electron ferromagnets was discussed in Refs. $5-7$, and the role of $e-e$ interactions in spin-wave damping was studied in Refs. 8 and 9 for spin-polarized liquid $\mathrm{He}^{3}$ and in Refs. 10 and 11 for two- and three-dimensional (3D) electron liquids, respectively. In this Rapid Communication, we present a unified semiphenomenological approach, which enables us to calculate on equal footing the contributions of disorder and $e-e$ interactions to the Gilbert damping parameter to order $q^{2}$. The main idea is to apply to the transverse spin fluctuations of a ferromagnet the method first introduced by Mermin ${ }^{12}$ for treating the effect of disorder on the dynamics of charge-density fluctuations in metals. ${ }^{13}$ Following this approach, we will show that the $q^{2}$ contribution to the damping in itinerant electron ferromagnets can be expressed in terms of the transverse spin conductivity, which in turn separates into a sum of disorder and $e-e$ terms.

A major technical advantage of this approach is that the ladder vertex corrections to the transverse spin conductivity vanish in the absence of SO interactions, making the diagrammatic calculation of this quantity a straightforward task. Thus we are able to provide explicit analytic expressions for the disorder and interaction contribution to the $q^{2}$ Gilbert damping to the lowest order in the strength of the interactions. This Rapid Communication connects and unifies different approaches and gives a rather complete and simple theory of $q^{2}$ damping. In particular, we find that for weak metallic ferromagnets the $q^{2}$ damping can be strongly enhanced by $e-e$ interactions, resulting in a value comparable to or larger than typical in the case of homogeneous damping. Therefore, we believe that the inclusion of a damping term proportional to $q^{2}$ in the phenomenological LandauLifshitz equation of motion for the magnetization ${ }^{14}$ is a potentially important modification of the theory in strongly inhomogeneous situations, such as current-driven nanomagnets $^{2}$ and the ferromagnetic domain-wall motion. ${ }^{15,16}$

\section{PHENOMENOLOGICAL APPROACH}

In Ref. 12, Mermin constructed the density-density response function of an electron gas in the presence of impurities through the use of a local drift-diffusion equation, whereby the gradient of the external potential is cancelled, in equilibrium, by an opposite gradient of the local chemical potential. In diagrammatic language, the effect of the local chemical potential corresponds to the inclusion of the vertex correction in the calculation of the density-density response function. Here, we use a similar approach to obtain the transverse spin susceptibility of an itinerant electron ferromagnet, modeled as an electron gas whose equilibrium magnetization is along the $z$ axis.

Before proceeding we need to clarify a delicate point. The homogeneous electron gas is not spontaneously ferromagnetic at the densities that are relevant for ordinary magnetic systems. ${ }^{13}$ In order to produce the desired equilibrium magnetization, we must therefore impose a static fictitious field $B_{0}$. Physically, $B_{0}$ is the "exchange" field $B_{\mathrm{ex}}$ plus any external/applied magnetic field $B_{0}^{\text {app }}$ which may be addition- 
ally present. Therefore, in order to calculate the transverse spin susceptibility we must take into account the fact that the exchange field associated with a uniform magnetization is parallel to the magnetization and changes direction when the latter does. As a result, the actual susceptibility $\chi_{a b}(\mathbf{q}, \omega)$ differs from the susceptibility calculated at constant $B_{0}$, which we denote by $\tilde{\chi}_{a b}(\mathbf{q}, \omega)$, according to the well-known relation $^{11}$

$$
\chi_{a b}^{-1}(\mathbf{q}, \omega)=\widetilde{\chi}_{a b}^{-1}(\mathbf{q}, \omega)-\frac{\omega_{\mathrm{ex}}}{M_{0}} \delta_{a b} .
$$

Here, $M_{0}$ is the equilibrium magnetization (assumed to point along the $z$ axis) and $\omega_{\mathrm{ex}}=\gamma B_{\mathrm{ex}}$ (where $\gamma$ is the gyromagnetic ratio) is the precession frequency associated with the exchange field. $\delta_{a b}$ is the Kronecker delta. The indices $a$ and $b$ denote directions ( $x$ or $y$ ) perpendicular to the equilibrium magnetization and $\mathbf{q}$ and $\omega$ are the wave vector and the frequency of the external perturbation. Here we focus solely on the calculation of the response function $\tilde{\chi}$ because term $\omega_{\mathrm{ex}} \delta_{a b} / M_{0}$ does not contribute to Gilbert damping. We do not include the effects of exchange and external fields on the orbital motion of the electrons.

The generalized continuity equation for the Fourier component of the transverse spin density $M_{a}$ in the direction $a(x$ or $y$ ) at wave vector $\mathbf{q}$ and frequency $\omega$ is

$$
\begin{aligned}
-i \omega M_{a}(\mathbf{q}, \omega)= & -i \gamma \mathbf{q} \cdot \mathbf{j}_{a}(\mathbf{q}, \omega)-\omega_{0} \epsilon_{a b} M_{b}(\mathbf{q}, \omega) \\
& +\gamma M_{0} \epsilon_{a b} B_{b}^{\mathrm{app}}(\mathbf{q}, \omega),
\end{aligned}
$$

where $B_{a}^{\text {app }}(\mathbf{q}, \omega)$ is the transverse external magnetic field driving the magnetization and $\omega_{0}$ is the precessional frequency associated with a static magnetic field $B_{0}$ (including exchange contribution) in the $z$ direction. $\mathbf{j}_{a}$ is the $a$ th component of the transverse spin-current-density tensor and we set $\hbar=1$ throughout. The transverse Levi-Civita tensor $\epsilon_{a b}$ has components $\epsilon_{x x}=\epsilon_{y y}=0, \epsilon_{x y}=-\epsilon_{y x}=1$, and the summation over repeated indices is always implied.

The transverse spin current is proportional to the gradient of the effective magnetic field, which plays the role analogous to the electrochemical potential, and the equation that expresses this proportionality is the analog of the driftdiffusion equation of the ordinary charge transport theory,

$$
\mathbf{j}_{a}(\mathbf{q}, \omega)=i \mathbf{q} \sigma_{\perp}\left[\gamma B_{a}^{\mathrm{app}}(q, \omega)-\frac{M_{a}(\mathbf{q}, \omega)}{\tilde{\chi}_{\perp}}\right],
$$

where $\sigma_{\perp}\left(=\sigma_{x x}\right.$ or $\left.\sigma_{y y}\right)$ is the transverse dc (i.e., $\left.\omega=0\right)$ spin conductivity and $\tilde{\chi}_{\perp}=M_{0} / \omega_{0}$ is the static transverse spin susceptibility in the $q \rightarrow 0$ limit. ${ }^{17}$ Just as in the ordinary driftdiffusion theory, the first term on the right-hand side of Eq. (3) is a "drift current" and the second is a "diffusion current," with the two canceling out exactly in the static limit (for $q$ $\rightarrow 0$ ), due to the relation $M_{a}(0,0)=\gamma \tilde{\chi}_{\perp} B_{a}^{\text {app }}(0,0)$. Combining Eqs. (2) and (3) gives the following equation for the transverse magnetization dynamics:

$$
\begin{gathered}
\left(-i \omega \delta_{a b}+\frac{\gamma \sigma_{\perp} q^{2}}{\tilde{\chi}_{\perp}} \delta_{a b}+\omega_{0} \epsilon_{a b}\right) M_{b} \\
=\left(M_{0} \epsilon_{a b}+\gamma \sigma_{\perp} q^{2} \delta_{a b}\right) \gamma B_{b}^{\mathrm{app}},
\end{gathered}
$$

which is most easily solved by transforming to the circularly polarized components $M_{ \pm}=M_{x} \pm i M_{y}$, in which the LeviCivita tensor becomes diagonal, with eigenvalues $\pm i$. Solving in the "+" channel, we get

$$
M_{+}=\gamma \tilde{\chi}_{+-} B_{+}^{\mathrm{app}}=\frac{M_{0}-i \gamma \sigma_{\perp} q^{2}}{\omega_{0}-\omega-i \gamma \sigma_{\perp} q^{2} \omega_{0} / M_{0}} \gamma B_{+}^{\mathrm{app}},
$$

from which we obtain to the leading order in $\omega$ and $q^{2}$

$$
\tilde{\chi}_{+-}(q, \omega) \simeq \frac{M_{0}}{\omega_{0}}\left(1+\frac{\omega}{\omega_{0}}\right)+i \omega \frac{\gamma \sigma_{\perp} q^{2}}{\omega_{0}^{2}} .
$$

The higher-order terms in this expansion cannot be legitimately retained within the accuracy of the present approximation. We also disregard the $q^{2}$ correction to the static susceptibility, since in making the Mermin ansatz (3) we are omitting the equilibrium spin currents responsible for the latter. Equation (6), however, is perfectly adequate for our purpose, since it allows us to identify the $q^{2}$ contribution to the Gilbert damping,

$$
\alpha=\frac{\omega_{0}^{2}}{M_{0}} \lim _{\omega \rightarrow 0} \frac{\Im m \tilde{\chi}_{+-}(q, \omega)}{\omega}=\frac{\gamma \sigma_{\perp} q^{2}}{M_{0}} .
$$

Therefore, the Gilbert damping can be calculated from the dc transverse spin conductivity $\sigma_{\perp}$, which in turn can be computed from the zero-frequency limit of the transverse spincurrent-spin-current response function,

$$
\sigma_{\perp}=-\frac{1}{m_{*}^{2} \mathcal{V}} \lim _{\omega \rightarrow 0} \frac{\Im m\left\langle\left\langle\sum_{i=1}^{N} \hat{S}_{i a} \hat{p}_{i a} ; \sum_{i=1}^{N} \hat{S}_{i a} \hat{p}_{i a}\right\rangle\right\rangle_{\omega}}{\omega},
$$

where $\hat{S}_{i a}$ is the $x$ or $y$ component of the spin operator for the $i$ th electron, $\hat{p}_{i a}$ is the corresponding component of the momentum operator, $m_{*}$ is the effective electron mass, $\mathcal{V}$ is the system volume, $N$ is the total electron number, and $\langle\langle\hat{A} ; \hat{B}\rangle\rangle_{\omega}$ represents the retarded linear-response function for the expectation value of an observable $\hat{A}$ under the action of a field that couples linearly to an observable $\hat{B}$. Both disorder and $e-e$ interaction contributions can be systematically included in the calculation of the spin-current-spin-current response function. In the absence of spin-orbit and $e-e$ interactions, the ladder vertex corrections to the conductivity are absent and calculation of $\sigma_{\perp}$ reduces to the calculation of a single bubble with Green's functions,

$$
G_{\uparrow, \downarrow}(\mathbf{p}, \omega)=\frac{1}{\omega-\varepsilon_{\mathbf{p}}+\varepsilon_{F} \pm \omega_{0} / 2+i / 2 \tau_{\uparrow, \downarrow}},
$$

where the scattering time $\tau_{s}$ in general depends on the spin band index $s=\uparrow, \downarrow$. In the Born approximation, the scattering rate is proportional to the electron density of states, and we can write $\tau_{\uparrow, \downarrow}=\tau \nu / \nu_{\uparrow, \downarrow}$, where $\nu_{s}$ is the spin- $s$ density of states and $\nu=\left(\nu_{\uparrow}+\nu_{\downarrow}\right) / 2$. $\tau$ parametrizes the strength of the 
disorder scattering. A standard calculation then leads to the following result:

$$
\sigma_{\perp}^{\mathrm{dis}}=\frac{v_{F \uparrow}^{2}+\nu_{F \downarrow}^{2}}{6\left(\nu_{\downarrow}^{-1}+\nu_{\uparrow}^{-1}\right)} \frac{1}{\omega_{0}^{2} \tau} .
$$

This, inserted in Eq. (7), gives a Gilbert damping parameter in full agreement with what we have also calculated from a direct diagrammatic evaluation of the transverse spin susceptibility, i.e., spin-density-spin-density correlation function. From now on, we shall simplify the notation by introducing a transverse spin-relaxation time,

$$
\frac{1}{\tau_{\perp}^{\mathrm{dis}}}=\frac{4 E_{F \uparrow}+E_{F \downarrow}}{3 n\left(\nu_{\downarrow}^{-1}+\nu_{\uparrow}^{-1}\right)} \frac{1}{\tau},
$$

where $E_{F s}=m_{*} v_{F s}^{2} / 2$ is the Fermi energy for spin-s electrons and $n$ is the total electron density. In this notation, the dc transverse spin conductivity takes the form

$$
\sigma_{\perp}^{\mathrm{dis}}=\frac{n}{4 m_{*} \omega_{0}^{2}} \frac{1}{\tau_{\perp}^{\mathrm{dis}}} .
$$

\section{ELECTRON-ELECTRON INTERACTIONS}

One of the attractive features of the approach based on Eq. (8) is the ease with which $e$-e interactions can be included. In the weak-coupling limit, the contributions of disorder and $e-e$ interactions to the transverse spin conductivity are simply additive. We can see this by using twice the equation of motion for the spin-current-spin-current response function. This leads to an expression for the transverse spin conductivity [Eq. (8)] in terms of the low-frequency spinforce-spin-force response function,

$$
\sigma_{\perp}=-\frac{1}{m_{*}^{2} \omega_{0}^{2} \mathcal{V}} \lim _{\omega \rightarrow 0} \frac{\Im m\left\langle\left\langle\sum_{i} \hat{S}_{i a} \hat{F}_{i a} ; \sum_{i} \hat{S}_{i a} \hat{F}_{i a}\right\rangle\right\rangle_{\omega}}{\omega} .
$$

Here, $\hat{F}_{i a}=\dot{\hat{p}}_{i a}$ is the time derivative of the momentum operator, i.e., the operator of the force on the $i$ th electron. The total force is the sum of electron-impurity and $e-e$ interaction forces. Each of them, separately, gives a contribution of or$\operatorname{der}\left|v_{e i}\right|^{2}$ and $\left|v_{e e}\right|^{2}$, where $v_{e i}$ and $v_{e e}$ are matrix elements of the electron-impurity and $e-e$ interactions, respectively, while cross terms are of higher order, e.g., $v_{e e}\left|v_{e i}\right|^{2}$. Thus, the two interactions give additive contributions to the conductivity. In Ref. 18, a phenomenological equation of motion was used to find the spin current in a system with disorder and longitudinal spin-Coulomb drag coefficient. We can use a similar approach to obtain transverse spin currents with transverse spin-Coulomb drag coefficient $1 / \tau_{\perp}^{e e}$. In the circularly polarized basis,

$$
i\left(\omega \mp \omega_{0}\right) j_{ \pm}=-\frac{n E_{ \pm}}{4 m_{*}}+\frac{j_{ \pm}}{\tau_{\perp}^{\mathrm{dis}}}+\frac{j_{ \pm}}{\tau_{\perp}^{e e}},
$$

and correspondingly the spin conductivities are

$$
\sigma_{ \pm}=\frac{n}{4 m_{*}}-\left(\omega \mp \omega_{0}\right) i+1 / \tau_{\perp}^{\mathrm{dis}}+1 / \tau_{\perp}^{e e} .
$$

In the dc limit, this gives

$$
\sigma_{\perp}(0)=\frac{\sigma_{+}+\sigma_{-}}{2}=\frac{n}{4 m_{*}} \frac{1 / \tau_{\perp}^{\mathrm{dis}}+1 / \tau_{\perp}^{e e}}{\omega_{0}^{2}+\left(1 / \tau_{\perp}^{\mathrm{dis}}+1 / \tau_{\perp}^{e e}\right)^{2}} .
$$

Using Eq. (16), an identification of the $e-e$ contribution is possible in a perturbative regime where $1 / \tau_{\perp}^{e e}$ and $1 / \tau_{\perp}^{\mathrm{dis}}$ $\ll \omega_{0}$, leading to the following formula:

$$
\sigma_{\perp}=\frac{n}{4 m_{*} \omega_{0}^{2}}\left(\frac{1}{\tau_{\perp}^{\mathrm{dis}}}+\frac{1}{\tau_{\perp}^{e e}}\right) .
$$

Comparison with Eq. (13) enables us to immediately identify the microscopic expressions for the two scattering rates. For the disorder contribution, we recover what we already knew, i.e., Eq. (11). For the $e-e$ interaction contribution, we obtain

$$
\frac{1}{\tau_{\perp}^{e e}}=-\frac{4}{n m_{*} \mathcal{V}_{\omega \rightarrow 0}} \lim _{\omega} \frac{\Im m\left\langle\left\langle\sum_{i} \hat{S}_{i a} \hat{F}_{i a}^{C} ; \sum_{i} \hat{S}_{i a} \hat{F}_{i a}^{C}\right\rangle\right\rangle_{\omega}}{\omega},
$$

where $F^{C}$ is just the Coulomb force, and the force-force correlation function is evaluated in the absence of disorder. The correlation function in Eq. (18) is proportional to the function $F_{+-}(\omega)$ which appeared in Ref. 11 [Eqs. (18) and (19)] in a direct calculation of the transverse spin susceptibility. Making use of the analytic result for $\mathfrak{I} m F_{+-}(\omega)$ presented in Eqs. (21) and (24) of that paper we obtain

$$
\frac{1}{\tau_{\perp}^{e e}}=\Gamma(p) \frac{8 \alpha_{0}}{27} \frac{T^{2} r_{s}^{4} m_{*} a_{*}^{2} k_{B}^{2}}{(1+p)^{1 / 3}}
$$

where $T$ is the temperature, $p=\left(n_{\uparrow}-n_{\downarrow}\right) / n$ is the degree of spin polarization, $a_{*}$ is the effective Bohr radius, $r_{s}$ is the dimensionless Wigner-Seitz radius, $\alpha_{0}=(4 / 9 \pi)^{1 / 3}$, and $\Gamma(p)$ - a dimensionless function of the polarization $p$-is defined by Eq. (23) of Ref. 11. This result is valid to second order in the Coulomb interaction. Collecting our results, we finally obtain a full expression for the $q^{2}$ Gilbert damping parameter,

$$
\alpha=\frac{\gamma n q^{2}}{4 m_{*} M_{0}} \frac{1 / \tau_{\perp}^{\mathrm{dis}}+1 / \tau_{\perp}^{e e}}{\omega_{0}^{2}+\left(1 / \tau_{\perp}^{\mathrm{dis}}+1 / \tau_{\perp}^{e e}\right)^{2}} .
$$

One of the salient features of Eq. (20) is that it scales as the total scattering rate in the weak disorder and $e-e$ interaction limit, while it scales as the scattering time in the opposite limit. The approximate formula for the Gilbert damping in the more interesting weak-scattering/strong-ferromagnet regime is

$$
\alpha=\frac{\gamma n q^{2}}{4 m_{*} \omega_{0}^{2} M_{0}}\left(\frac{1}{\tau_{\perp}^{\text {dis }}}+\frac{1}{\tau_{\perp}^{e e}}\right),
$$

while in the opposite limit, i.e., for $\omega_{0} \ll 1 / \tau_{\perp}^{\mathrm{dis}}, 1 / \tau_{\perp}^{e e}$,

$$
\alpha=\frac{\gamma n q^{2}}{4 m_{*} M_{0}}\left(\frac{1}{\tau_{\perp}^{\mathrm{dis}}}+\frac{1}{\tau_{\perp}^{e e}}\right)^{-1} .
$$

Equation (20) agrees with the result of Singh and Tesanovic ${ }^{6}$ on the spin-wave linewidth as a function of the disorder strength and $\omega_{0}$. However, Eq. (20) also describes the influence of $e-e$ correlations on the Gilbert damping. A comparison of the scattering rates originating from disorder and $e-e$ 


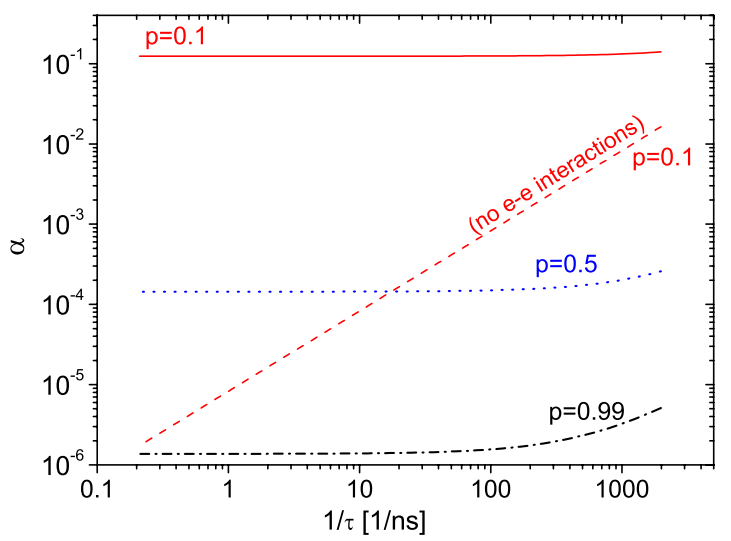

FIG. 1. (Color online) The Gilbert damping $\alpha$ as a function of the disorder scattering rate $1 / \tau$. The red (solid) line shows the Gilbert damping for polarization $p=0.1$ in the presence of the $e-e$ and disorder scattering, while the dashed line does not include the $e-e$ scattering. The blue (dotted) and black (dash-dotted) lines show Gilbert damping for $p=0.5$ and $p=0.99$, respectively. We took $q=0.1 k_{F}, T=54 \mathrm{~K}, \omega_{0}=E_{F}\left[(1+p)^{2 / 3}-(1-p)^{2 / 3}\right], M_{0}=\gamma p n / 2$, $m_{*}=m_{e}, n=1.4 \times 10^{21} \mathrm{~cm}^{-3}, r_{s}=5$, and $a_{*}=2 a_{0}$.

interactions shows that the latter is important and can be comparable or even greater than the disorder contribution for high-mobility and/or low-density 3D metallic samples. Figure 1 shows the behavior of the Gilbert damping as a function of the disorder scattering rate. One can see that the $e-e$ scattering strongly enhances the Gilbert damping for small polarizations/weak ferromagnets [see the red (solid) line]. This stems from the fact that $1 / \tau_{\perp}^{\mathrm{dis}}$ is proportional to $1 / \tau$ and independent of polarization for small polarizations, while $1 / \tau_{\perp}^{e e}$ is enhanced by a large prefactor $\Gamma(p)=2 \lambda /\left(1-\lambda^{2}\right)$ $+(1 / 2) \ln [(1+\lambda) /(1-\lambda)]$, where $\lambda=(1-p)^{1 / 3} /(1+p)^{1 / 3}$. On the other hand, for strong polarizations (dotted and dashdotted lines in Fig. 1), the disorder dominates in a broad range of $1 / \tau$ and the inhomogenous contribution to the Gilbert damping is rather small. Finally, we note that our calculation of the $e-e$ interaction contribution to the Gilbert damp- ing is valid under the assumption of $\hbar \omega \ll k_{B} T$ (which is certainly the case if $\omega=0$ ). More generally, as follows from Eqs. (21) and (22) of Ref. 11, a finite frequency $\omega$ can be included through the replacement $\left(2 \pi k_{B} T\right)^{2} \rightarrow\left(2 \pi k_{B} T\right)^{2}$ $+(\hbar \omega)^{2}$ in Eq. (19). Thus $1 / \tau_{\perp}^{e e}$ is proportional to the scattering rate of quasiparticles near the Fermi level, and our damping constant in the clean limit becomes qualitatively similar to the damping parameter obtained by Mineev ${ }^{9}$ for $\omega$ corresponding to the spin-wave resonance condition in some external magnetic field (which in practice is much smaller than the ferromagnetic exchange splitting $\omega_{0}$ ).

\section{SUMMARY}

We have presented a unified theory of the Gilbert damping in itinerant electron ferromagnets at the order $q^{2}$, including $e-e$ interactions and disorder on equal footing. For the inhomogeneous dynamics $(q \neq 0)$, these processes add to a $q=0$ damping contribution that is governed by magnetic disorder and/or spin-orbit interactions. We have shown that the calculation of the Gilbert damping can be formulated in the language of the spin conductivity, which takes an intuitive Matthiessen form with the disorder and interaction contributions being simply additive. It is still a common practice, e.g., in the micromagnetic calculations of spin-wave dispersions and linewidths, to use a Gilbert damping parameter independent of $q$. However, such calculations are often at odds with experiments on the quantitative side, particularly where the linewidth is concerned. ${ }^{2}$ We suggest that the inclusion of the $q^{2}$ damping (as well as the associated magnetic noise) may help in reconciling theoretical calculations with experiments.

\section{ACKNOWLEDGMENTS}

This work was supported in part by the NSF under Grants No. DMR-0313681 and No. DMR-0705460 as well as Fordham Research Grant. Y.T. thanks A. Brataas and G. E. W. Bauer for useful discussions.

\footnotetext{
*hankiewicz@fordham.edu

${ }^{1}$ Y. Tserkovnyak, A. Brataas, G. E. Bauer, and B. I. Halperin, Rev. Mod. Phys. 77, 1375 (2005).

${ }^{2}$ I. N. Krivorotov, D. V. Berkov, N. L. Gorn, N. C. Emley, J. C. Sankey, D. C. Ralph, and R. A. Buhrman, Phys. Rev. B 76, 024418 (2007).

${ }^{3}$ T. L. Gilbert, IEEE Trans. Magn. 40, 3443 (2004).

${ }^{4}$ E. M. Hankiewicz, G. Vignale, and Y. Tserkovnyak, Phys. Rev. B 75, 174434 (2007).

${ }^{5}$ A. Singh, Phys. Rev. B 39, 505 (1989).

${ }^{6}$ A. Singh and Z. Tesanovic, Phys. Rev. B 39, 7284 (1989).

${ }^{7}$ V. L. Safonov and H. N. Bertram, Phys. Rev. B 61, R14893 (2000).

${ }^{8}$ V. P. Silin, Sov. Phys. JETP 6, 945 (1958).

${ }^{9}$ V. P. Mineev, Phys. Rev. B 69, 144429 (2004).

${ }^{10}$ Y. Takahashi, K. Shizume, and N. Masuhara, Phys. Rev. B 60, 4856 (1999)

${ }^{11}$ Z. Qian and G. Vignale, Phys. Rev. Lett. 88, 056404 (2002).
}

${ }^{12}$ N. D. Mermin, Phys. Rev. B 1, 2362 (1970).

${ }^{13}$ G. F. Giuliani and G. Vignale, Quantum Theory of the Electron Liquid (Cambridge University Press, Cambridge, UK, 2005).

${ }^{14}$ E. M. Lifshitz and L. P. Pitaevskii, Statistical Physics, Part 2, Course of Theoretical Physics Vol. 9, 3rd ed. (Pergamon, Oxford, 1980).

${ }^{15}$ Y. Tserkovnyak, A. Brataas, and G. E. Bauer, J. Magn. Magn. Mater. 320, 1282 (2008), and references therein.

${ }^{16}$ In ferromagnets which nonuniformities are beyond the linearized spin waves, there is a nonlinear $q^{2}$ contribution to damping [see J. Foros, A. Brataas, Y. Tserkovnyak, and G. E. W. Bauer, arXiv:0803.2175 (unpublished)] which has a different physical origin, related to the longitudinal spin-current fluctuations.

${ }^{17}$ Although both $\sigma_{\perp}$ and $\tilde{\chi}_{\perp}$ are in principle tensors in transverse spin space, they are proportional to $\delta_{a b}$ in axially symmetric systems; hence we use scalar notation.

${ }^{18}$ I. D'Amico and G. Vignale, Phys. Rev. B 62, 4853 (2000). 